\title{
Preparation of Mono- and Bis(germyl)nitrilimines from Germylenes and Stannyl Diazo Derivatives
}

\author{
Christian Leue, ${ }^{\dagger}$ Régis Réau, ${ }^{\ddagger}$ Beate Neumann, ${ }^{\dagger}$ Hans-Georg Stammler, ${ }^{\dagger}$ \\ Peter Jutzi, ${ }^{*}, \dagger$ and Guy Bertrand ${ }^{*,+}$
}

Fakultät für Chemie der Universität Bielefeld, D-4800 Bielefeld 1, Germany, and Laboratoire de Chimie de Coordination du CNRS, Université Paul Sabatier, 205 route de Narbonne, 31077 Toulouse Cédex, France

Received November 17, $1993^{\circledR}$

Summary: (Silyl)(germyl)- and bis(germyl)nitrilimines 2 and 5 are obtained by adding [bis(trimethylsilyl)methyl] (pentamethylcyclopentadienyl)germylene (1) to (triisopropylsilyl)(trimethylstannyl)diazomethane and bis(trimethylstannyl)diazomethane, respectively; the reaction'mechanism is discussed.

In the last few years, we have shown that, using the right set of substituents, nitrilimines can exist as stable compounds at room temperature. ${ }^{1}$ Nitrilimines have a bent allenic structure, ${ }^{2}$ except those possessing substituents with accessible vacant orbitals. In the latter case, nitrilimines have a propargylic type structure, ${ }^{3,4}$ and the interaction of the CNN skeleton with the heteroatom substituents induces a shortening of the heteroatomnitrogen or heteroatom-carbon bond lengths compared to classical single bonds; the $N$-phosphonio-3a and $C$-borylnitrilimines ${ }^{3 b}$ are typical examples. Therefore, it was tempting to prepare nitrilimines featuring germanediyl moieties, in order to study the multiple-bond character of the nitrogen-or/and carbon-germanium bond (Chart 1). Stannyl diazo derivatives react with a variety of electrophiles, leading to nitrilimines. ${ }^{2 b, 5}$ On the other hand, pentamethylcyclopentadienyl $\left(\mathrm{Cp}^{*}\right)$ substituted germylenes are known to undergo nucleophilic substitutions. ${ }^{6}$ Here we report the surprising results observed in the reaction of [bis(trimethylsilyl)methyl](pentamethylcyclopentadienyl)germylene (1) ${ }^{6 \mathrm{a}}$ with (triisopropylsilyl)(trimethylstannyl)diazomethane $e^{5}$ and bis(trimethylstannyl)diazomethane. ${ }^{7}$

(Triisopropylsilyl)(trimethylstannyl)diazomethane reacted at room temperature in a THF solution with 1 equiv of 1 , leading, after workup, to a pale yellow oil identified as a nitrilimine ${ }^{8}$ on the basis of ${ }^{14} \mathrm{~N} \mathrm{NMR}(\delta-183(\mathrm{CNN}))^{9}$ and ${ }^{13} \mathrm{C}$ NMR ( $\left.\delta 49.85(\mathrm{CNN})\right)$ data and the strong and broad absorption in the IR spectrum at $2076 \mathrm{~cm}^{-1}$.

$\uparrow$ Universität Bielefeld

t Université Paul Sabatier.

- Abstract published in Advance ACS Abstracts, January 15, 1994.

(1) For a review see: Bertrand, G.; Wentrup, K. Angew. Chem., Int. Ed. Engl., in press.

(2) (a) Granier, M.; Baceiredo, A.; Dartiguenave, Y.; Menu, M. J.; Bertrand, G. J. Am. Chem. Soc. 1990, 112,6277. (b) Réau, R.; Veneziani, G.; Dahan, F.; Bertrand, G. Angew. Chem., Int. Ed. Engl. 1992, 31, 439.

(3) (a) Granier, M.; Baceiredo, A.; Bertrand, G.; Huch, V.; Veith, M. Inorg. Chem. 1991, 30, 1161. (b) Arthur, M. P.; Baceiredo, A.; Bertrand, G. Synthesis 1992, 43 .

(4) (a) Wong, M. W.; Wentrup, K. J. Am. Chem. Soc. 1993, 115, 7743. (b) Wong, M. W.; Wentrup, K. To be submitted for publication.

(5) Réau, R.; Veneziani, G.; Bertrand, G.J. Am. Chem. Soc. 1992, 114, 6059 .

(6) (a) Jutzi, P.; Becker, A.; Leue, C.; Stammler, H. G.; Neumann, B. Organometallics 1991, 10, 3838. (b) Jutzi, P.; Becker, A.; Stammler, H. G.; Neumann, B. Organometallics 1991, 10, 1647. 2954.

(7) Lappert, M. F.; Lorberth, J.; Poland, J. S. J. Chem. Soc. A 1970,

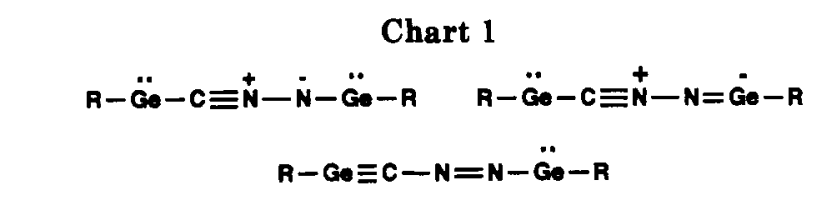

Scheme 1
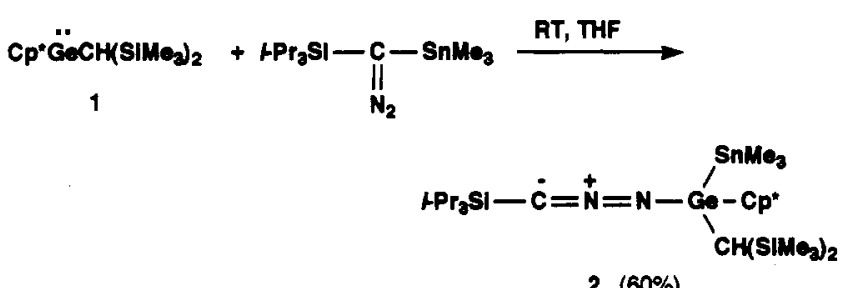

$C p^{*}=C_{5} M e_{5}$

Surprisingly, NMR data also revealed the presence of a trimethylstannyl group $\left({ }^{1} \mathrm{H}\right.$ NMR $\delta 0.48\left(J_{117} \mathrm{SnH}=47.2\right.$ $\left.\mathrm{Hz}, J_{119} \mathrm{SnH}=49.2 \mathrm{~Hz}, 9 \mathrm{H}\right) ;{ }^{13} \mathrm{C}$ NMR $\delta-4.28\left(J_{117_{\mathrm{SnH}}}=\right.$ $\left.\left.244.2 \mathrm{~Hz}, J_{119 \mathrm{SnH}}=255.6 \mathrm{~Hz}\right) ;{ }^{119} \mathrm{Sn} \mathrm{NMR} \delta-77.88\right)$ and also a fluxional $\mathrm{Cp}^{*}$ substituent $\left({ }^{1} \mathrm{H}\right.$ NMR $\delta 1.85(15 \mathrm{H})$;

(8) 2: Neat (triisopropylsilyl)(trimethylstannyl)diazomethane $(0.824$ $\mathrm{g}, 2.3 \mathrm{mmol}$ ) was added at room temperature to a THF solution $(5 \mathrm{~mL})$ of $1(0.838 \mathrm{~g}, 2.3 \mathrm{mmol})$. After the mixture was stirred for $1.5 \mathrm{~h}$ at room temperature, the solvent was removed under vacuum. The pale yellow oily nitrilimine 2 was washed three times with acetonitrile $(5 \mathrm{~mL})$ and dried in vacuo $(1.00 \mathrm{~g}, 60 \%$ yield $):$ bp $104^{\circ} \mathrm{C}\left(5 \times 10^{-2} \mathrm{mmHg}\right) ;{ }^{1} \mathrm{H}$ NMR $\left(\mathrm{C}_{6} \mathrm{D}_{6}\right) \delta-0.07,-0.06\left(\mathrm{~s}, 1 \mathrm{H}, \mathrm{CHSiCH}_{3}\right), 0.13,0.39\left(\mathrm{~s}, 18 \mathrm{H}, \mathrm{SiCH}_{3}\right), 0.48$ $\left(\mathrm{s}, J_{117 \mathrm{SnH}}=47.2 \mathrm{~Hz}, J_{118 \mathrm{gnH}}=49.2 \mathrm{~Hz}, 9 \mathrm{H}, \mathrm{SnCH}_{3}\right), 1.08(\mathrm{~m}, 21 \mathrm{H}$, $\mathrm{SiCHCH}$ ), 1.85 (broad s, $\left.15 \mathrm{H}, \mathrm{CpCH}_{3}\right) ;{ }^{13} \mathrm{C} \mathrm{NMR}\left(\mathrm{C}_{6} \mathrm{D}_{6}\right) \delta-4.28(\mathrm{~s}$ $\left.J_{117}{ }_{\mathrm{SnC}}=244.2 \mathrm{~Hz}, J_{11 \mathrm{BgC}_{\mathrm{SC}}}=255.6 \mathrm{~Hz}, \mathrm{SnCH}_{3}\right), 3.94,4.72\left(\mathrm{~s}, \mathrm{SiCH}_{3}\right), 12.92$ $(\mathrm{s}, \mathrm{SiCHCH})_{3}, 13.20$ (broad s, $\left.\mathrm{CpCH}_{3}\right), 19.21\left(\mathrm{~s}, \mathrm{SiCHCH}_{3}\right), 49.85(\mathrm{~s}, \mathrm{CNN})$, 135.56 (broad $\mathrm{s}, \mathrm{Cp}$ ), $\mathrm{CHSiCH}_{3}$ was not observed, probably hidden by $\mathrm{SiCH}_{3} ;{ }^{14} \mathrm{~N} \mathrm{NMR}\left(\mathrm{C}_{6} \mathrm{D}_{6}\right) \delta-183\left(\nu_{1 / 2}=240 \mathrm{~Hz}, \mathrm{CNN}\right) ;{ }^{119} \mathrm{Sn} \mathrm{NMR}\left(\mathrm{C}_{6} \mathrm{D}_{6}\right)$ $\delta-77.88 ;{ }^{29} \mathrm{Si} \mathrm{NMR}\left(\mathrm{C}_{6} \mathrm{D}_{6}\right) \delta-0.6,-0.1,0.9 ; \mathrm{IR}\left(\mathrm{THF}, \nu\left(\mathrm{cm}^{-1}\right)\right) 2076(\mathrm{CNN})$ Anal. Calcd for $\mathrm{C}_{30} \mathrm{H}_{64} \mathrm{GeN}_{2} \mathrm{Si}_{3} \mathrm{Sn}$ : $\mathrm{C}, 49.46 ; \mathrm{H}, 8.85 ; \mathrm{N}, 3.84$. Found: $\mathrm{C}$, $49.49 ; \mathrm{H}, 8.80 ; \mathrm{N}, 3.79$. 5: The mixture of diastereomeric nitrilimines 5 was obtained as a pale yellow powder from a saturated THF solution at $-80^{\circ} \mathrm{C}\left(48 \%\right.$ yield): ${ }^{1} \mathrm{H}$ NMR $\left(\mathrm{C}_{6} \mathrm{D}_{6}\right) \delta=-0.57,-0.55,-0.47,-0.45(8,2$ $\left.\mathrm{H}, \mathrm{CHSiCH}_{3}\right), 0.05,0.12\left(\mathrm{~s}, 18 \mathrm{H}, \mathrm{SiCH}_{3}\right), 0.37,0.38\left(\mathrm{~s}, J_{117 \mathrm{SnH}}=46.1 \mathrm{~Hz}\right.$, $J_{119 \mathrm{SnH}}=48.2 \mathrm{~Hz}, 9 \mathrm{H}, \mathrm{SnCH} H_{3}, 0.45,0.52\left(\mathrm{~s}, 18 \mathrm{H}, \mathrm{SiCH}_{3}\right), 0.63,0.64(\mathrm{~s}$, $\left.J_{11 \uparrow_{\mathrm{SnH}}}=46.2 \mathrm{~Hz}, J_{11 \mathrm{~g}_{\mathrm{gH}}}=48.1 \mathrm{~Hz}, 9 \mathrm{H}, \mathrm{SnCH}_{3}\right), 1.79($ broad s, $15 \mathrm{H}$ $\left.\mathrm{CpCH}_{3}\right), 1.90$ (broad s, $\left.15 \mathrm{H}, \mathrm{CpCH}_{3}\right) ;{ }^{13} \mathrm{C} \mathrm{NMR}\left(\mathrm{C}_{7} \mathrm{D}_{8}\right) \delta-5.22\left(\mathrm{~s}, J_{117} \mathrm{snC}\right.$ $\left.=250.1 \mathrm{~Hz}, J_{11 \mathrm{SnC}}=264.6 \mathrm{~Hz}, \mathrm{SnCH}_{3}\right),-4.28\left(\mathrm{~s}, J_{117_{\mathrm{SnC}}}=234.8 \mathrm{~Hz}, J_{11 \mathrm{SnC} \mathrm{C}}\right.$ $\left.=244.5 \mathrm{~Hz}, \mathrm{SnCH}{ }_{3}\right), 3.14,3.44,3.78,4.90\left(8, \mathrm{SiCH}_{3}\right), 12.40$ (broad s, $\mathrm{CpCH}_{3}$ ), $63.71\left(\mathrm{~s}, \mathrm{CNN}\right.$ ), 135.01 (broad s, $\mathrm{Cp}$ ), $\mathrm{CHSiCH}_{3}$ was not observed, probably hidden by $\mathrm{SiCH} \mathrm{CH}_{3}{ }^{14 \mathrm{~N}} \mathrm{NMR}\left(\mathrm{C}_{7} \mathrm{D}_{8}\right) \delta=-173\left(\nu_{1 / 2}=250 \mathrm{~Hz}, \mathrm{CNN}\right) ;{ }^{119 \mathrm{Sn}}$ NMR $\left(\mathrm{C}_{6} \mathrm{D}_{6}\right) \delta-83.12,-81.77,-67.09,-66.44 ;$ IR (ether, $\nu\left(\mathrm{cm}^{-1}\right)$ ) 2052 (CNN). Anal. Calcd for $\mathrm{C}_{41} \mathrm{H}_{86} \mathrm{Ge}_{2} \mathrm{~N}_{2} \mathrm{Si}_{4} \mathrm{Sn}_{2}: \mathrm{C}, 44.69 ; \mathrm{H}, 7.87 ; \mathrm{N}, 2.54$. Found: $\mathrm{C}, 44.71 ; \mathrm{H}, 7.79 ; \mathrm{N}, 2.55$. Single colorless crystals were grown from a $\mathrm{CH}_{2} \mathrm{Cl}_{2}$ solution at $-30^{\circ} \mathrm{C}$ : $\mathrm{mp} 136^{\circ} \mathrm{C}$. 7: Carbodiimide 7 was characterized in solution ( $40 \%$ spectroscopic yield): ${ }^{1} \mathrm{H} \mathrm{NMR}\left(\mathrm{C}_{6} \mathrm{D}_{6}\right) \delta$ $-0.15(\mathrm{~s}, 1 \mathrm{H}, \mathrm{CHSiCH})_{3}, 0.09,0.36(\mathrm{~s}, 18 \mathrm{H}, \mathrm{SiCH}), 0.42\left(\mathrm{~s}, J_{117 \mathrm{~S}_{\mathrm{H}}}=47.7\right.$ $\left.\mathrm{Hz}, J_{119 \mathrm{SnH}}=50.0 \mathrm{~Hz}, 9 \mathrm{H}, \mathrm{SnCH} H_{3}\right), 1.16(\mathrm{~m}, 21 \mathrm{H}, \mathrm{SiCHCH}), 1.82(\mathrm{broad}$ s, $15 \mathrm{H}, \mathrm{CpCH})_{3}{ }^{13} \mathrm{C} \mathrm{NMR}\left(\mathrm{C}_{6} \mathrm{D}_{6}\right) \delta-5.94\left(\mathrm{~s}, J_{117} \mathrm{SnC}=257.1 \mathrm{~Hz}, J_{11 \mathrm{SnC}}\right.$ $\left.=268.5 \mathrm{~Hz}, \mathrm{SnCH}_{3}\right) 3.65,4.33(\mathrm{~s}, \mathrm{SiCH}), 13.00\left(\right.$ broad s, $\left.\mathrm{CpCH}_{3}\right), 13.51$ $\left(\mathrm{s}, \mathrm{SiCHCH}{ }_{3}\right), 18.79$ (s, $\mathrm{SiCHCH}$ ), 126.41 (s, NCN), 136.10 (broad s, Cp), $\mathrm{CHSiCH}_{3}$ was not observed, probably hidden by $\mathrm{SiCH}{ }_{3} ;{ }^{119} \mathrm{Sn} \mathrm{NMR}\left(\mathrm{C}_{6} \mathrm{D}_{6}\right)$ $\delta-64.46$; IR (toluene, $\left.\nu\left(\mathrm{cm}^{-1}\right)\right) 2162(\mathrm{CNN}) ;{ }^{14} \mathrm{~N} \mathrm{NMR}\left(\mathrm{C}_{6} \mathrm{D}_{6}\right) \delta-365\left(\nu_{1 / 2}\right.$ $=390 \mathrm{~Hz}, \mathrm{NCN}$ ).

(9) Horchler von Locquenghien, K.; Réau, R.; Bertrand, G. J. Chem. Soc., Chem. Commun. 1991, 1192. 